\title{
Schistosome sirtuins as drug targets
}

The sirtuins form a superfamily of evolutionarily conserved $\mathrm{NAD}^{+}$-dependent protein $\mathrm{N}$ - $\varepsilon$-acyl-lysine (AcK) deacylases with roles in a variety of key cellular processes. Sirtuins have a broadly conserved overall structure with a catalytic site formed by a hydrophobic channel between the $\mathrm{NAD}^{+}$-binding Rossmann fold domain and a smaller $\mathrm{Zn}^{2+}$-binding domain. Schistosomes express five members of the sirtuin family and generic sirtuin inhibitors induce apoptosis and death in schistosome larvae, the disruption of adult worm pairs, inhibition of egg laying and damage to the male and female worm reproductive systems. Sirtuins in schistosomes and other parasitic flatworms present structural differences from their human orthologues that should allow the development of selective inhibitors that can be developed as drug leads.

\section{Schistosomiasis: strategies for drug discovery}

The treatment and control of schistosomiasis currently relies entirely on the use of the only available drug, praziquantel. Despite the undoubted success of the programs of mass treatment of school-age children in subSaharan Africa using this drug in reducing mortality and morbidity, notably through the Schistosomiasis Control Initiative [1], the potential for the development of resistance by the parasite [2] means that the development of new drugs is an absolute priority. The annotated genome sequences for the three major species of schistosome infecting humans [3-5] have provided an opportunity for the exploration of new strategies for drug discovery and we have chosen to pursue the targeting of schistosome epigenetic mechanisms [6]. The complex life cycle of the schistosomes, involving two hosts and four morphologically distinct forms, highlights the importance of a tight control of gene transcription during schistosome development, and the probable importance of epigenetic actors in this process. Moreover, these actors are increasingly targeted in other pathologies, notably in cancer, and the large libraries of compounds that have been identified can be exploited for test- ing against schistosomes, providing the basis for the development of lead compounds for antischistosome drug development. Here we focus on one family of such epigenetic actors, the sirtuins, and assess their potential as therapeutic targets in schistosomiasis.

\section{Why the boom in research on sirtuins?}

Initially categorized as class III histone deacetylases (HDAC), sirtuins are $\mathrm{NAD}^{+}-$ dependent protein $\mathrm{N}$ - - -acyl-lysine (AcK) deacylases that are phylogenetically and structurally distinct from the class I and II HDACs. Sirtuins are involved in a wide spectrum of biological functions: regulating transcriptional repression, cellular pathways, metabolism, aging, apoptosis and DNA damage responses [7,8]. They have also been linked to numerous human diseases [9]. Notably, it is now clear that sirtuins can either promote or repress tumor development and the discovery of inhibitors and activators of these enzymes gives the possibility of controlled intervention in either context, ensuring that sirtuins constitute a hot spot of research in this field [10]. In the case of parasitic pathogens, certain sirtuins have been shown to be essential to the survival and/or development
Julien Lancelot' ${ }^{1}$, Alejandro Cabezas-Cruz 1 , Stéphanie Caby', Martin Marek², Johan Schultz ${ }^{3}$, Christophe Romier², Wolfgang Sippl ${ }^{4}$, Manfred Jung $^{5}$ \& Raymond J Pierce ${ }^{*, 1}$ ${ }^{1}$ Center for Infection \& Immunity of Lille (CIIL), INSERM U1019 - CNRS UMR 8204, Université de Lille, Institut Pasteur de Lille, 1 rue Professeur Calmette, 59019 Lille Cedex, France

2Département de Biologie Structurale Intégrative, Institut de Génétique et Biologie Moléculaire et Cellulaire (IGBMC), Université de Strasbourg (UDS), CNRS, INSERM, 1 rue Laurent Fries, B.P. 10142, 67404 Illkirch Cedex, France ${ }^{3}$ Kancera $A B$, Karolinska Institutet Science Park, Banvaktsvägen 22, 17148 Solna, Sweden ${ }^{4}$ Institute of Pharmacy, Martin-LutherUniversität Halle-Wittenberg, WolfgangLangenbeck-Str. 4, 06120 Halle/Saale, Germany

${ }^{5}$ Institut für Pharmazeutische Wissenschaften, Albert-LudwigsUniversität Freiburg, Albertstraße 25, 79104 Freiburg, Germany *Author for correspondence: Tel.: + +33 320877783 Fax: +33 320877888 raymond.pierce@pasteur-lille.fr 


\section{Key term}

Sirtuin: $\mathrm{NAD}^{+}$-dependent protein deacylases, also categorized as class III histone deacetylases. Five orthologues of the seven human sirtuins are present in schistosomes.

of these organisms, determining their interest as drug targets.

Recently, the sirtuin family has been phylogenetically and molecularly characterized among all the kingdoms of life, and they were found in all species of Bacteria, Archea, Protists, Plants, Fungi, Ecdysozoa, Choanozoa, Echinodermata, Radiata, Lophotrochozoa and Chordata [11]. Importantly, from the structural point of view, sirtuins present a conserved and druggable $\mathrm{NAD}^{+}$and substrate pocket [12], allowing the use of inhibitors, that have been characterized in one organism, in distantly related organisms. At the same time sirtuins are different enough [12], to permit the design of species-specific inhibitors. All these properties make sirtuins ideal targets for drug discovery. Sirtuins have already been identified in important human and animal protozoan parasites [13] and recently they were described as 'emerging antiparasitic targets' [14]. However, in the literature, the sirtuins of important parasitic worms are underrepresented. Recently, our group identified members of the sirtuin family in the genome of the fluke Schistosoma mansoni and proposed their use as targets for antischistosomiasis drug development $[15,16]$. Herein, we review the structure, phylogeny and biological roles of sirtuins in relation to their interest as therapeutic targets in parasitic platyhelminths, and more particularly in schistosomes.

\section{Platyhelminthes present an evolutionarily conserved family of sirtuins}

Sirtuins are grouped into four main classes that contain all the metazoan representatives of this family, while a fifth group contains sirtuins from gram positive bacteria and the gram negative, hyperthermophile bacterium Thermotoga maritima [17], although groups I and IV are further subdivided and this organization was subsequently extended and consolidated [11]. It appears that the seven sirtuins (Sirt1-7) present in metazoans arose very early during animal evolution and that because of the importance of their functions, they have been conserved in all branches of the phylogenetic tree [11]. However, it is interesting to note that some organisms lack certain sirtuins, having only one or a few representatives of this family [11]. The distribution of sirtuins in parasitic Platyhelminthes is a good example of this 'selective lack of sirtuins' (Figure 1). For example, Schistosoma spp. lack Sirt3 and Sirt4; Clonorchis sinensis, the Chinese liver fluke, lacks Sirt6; and Echinococcus spp., lack Sirt4 and Sirt5. In sharp contrast, Sirt1 and Sirt2 seem to be ubiquitous. One could speculate that the parasitic life style would have made some sirtuins unnecessary, however, Schmidtea mediterranea, a free-living planarian lacks Sirt7. The presence of the seven sirtuins seems to be unrelated to the complexity of the organism. Thus, the seven sirtuins are present in at least one organism from Radiata (Nematostella vectensis, sea anemone) and Lophotrochozoa (Capitella capitata, a polychaete worm), although all ecdysozoan invertebrates seem to lack Sirt3. Most, but by no means all, vertebrates present the seven sirtuins. Exceptions are provided by the absence of Sirt1 and 7 in the zebrafish and of Sirt 2 in the pufferfish Tetraodon nigroviridis [11]. However, most unicellular organisms present only one or two sirtuins [1], and choanoflagellates (e.g., Monosiga brevicollis), considered to be the closest living relatives of metazoans [18], lack Sirt3 and Sirt5. It is considered that Sirt3 originated rather recently, as the Sirt3 group is present only in animal species, while Sirt5 is related to bacterial forms and is considered to be evolutionarily ancient [11]. Thus sirtuins have been lost or gained in eukaryotic genomes across evolution.

\section{Sirtuin cellular functions}

Sirtuins (Sirt1-7) share the highly conserved catalytic core but vary in subcellular localization, substrate specificity and function. In mammals Sirt1, Sirt 6 and Sirt7 localize to the nucleus, Sirt3, Sirt4 and Sirt5 are mitochondrial, while Sirt2 is predominantly cytoplasmic [19]. However, both Sirt2 and Sirt3 can also localize to the nucleus. Sirt2 shuttles to the nucleus during mitosis, where it can act as a histone deacetylase on H4K16 and H3K56 [20,21] and Sirt3 can shift to the nucleus under conditions of cellular stress [22]. In addition, Sirt5 activity is detectable in the cytoplasm [23] and Matsushita et al. [24] identified two isoforms of human Sirt5, with a shorter, predominately mitochondrial form and a longer isoform located in both the cytoplasm and mitochondria.

Although the subcellular localizations of schistosome sirtuins have not been determined experimentally, the presence (or absence) of signal sequences is indicative (Figure 2). However, although both Sirt 6 and Sirt7 have consensus nuclear export signals, they lack the nuclear localization signal, which is present at the C-terminal end of Sirt1 from S. mansoni. The corresponding signal is absent from the predicted Schistosoma japonicum Sirt1 peptide sequence, but the latter is probably incomplete at the C-terminal end. In contrast, a mitochondrial targeting peptide sequence is absent from $S$. mansoni Sirt5, but present in the $S$. japonicum orthologue, suggesting that the 


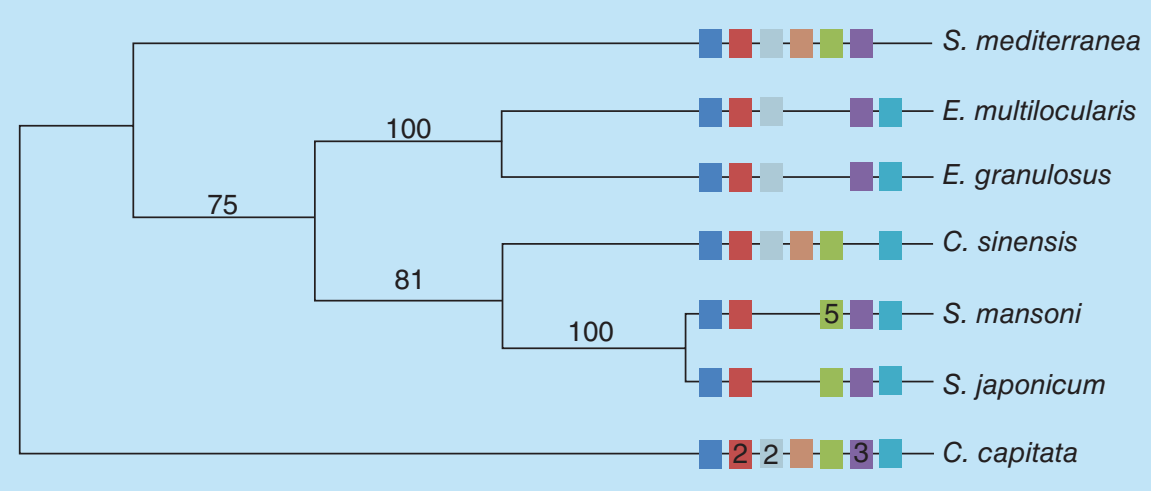

\begin{tabular}{|l|}
\hline Legend \\
Sirt 1 \\
Sirt 2 \\
Sirt 3 \\
Sirt 4 \\
Sirt 5 \\
Sirt 6 \\
Sirt 7 \\
\hline
\end{tabular}

Figure 1. Distribution of sirtuins amongst phylogenetically related Platyhelminthes. The figure displays a maximum likelihood phylogenetic tree build using $18 \mathrm{~S}$ rRNA gene from Schmidtea mediterranea (accession number: U31084), Echinococcus multilocularis (accession number: AB731634), Echinococcus granulosus (accession number: AB731639), Clonorchis sinensis (accession number: JF314770), Schistosoma mansoni (accession number: U65657), Schistosoma japonicum (accession number: AY157226) and Capitella capitata (accession number: U67323). The Sirtuins present in the different species are presented as colored squares as shown in the legend of the figure. The values on the internal branches represent bootstrap values (1000 bootstrap replicates).

sequences of the S. mansoni Sirt5 isoforms [16] should be re-examined.

The substrates and functions of human sirtuins have been the subject of recent, extensive reviews (e.g., [10,26]) and here we will only present a brief overview. Since the discovery of the role of Saccharomyces cerevisiae Sir2 in promoting longevity through increased gene dosage [27], followed by similar observations in Caenorhabditis elegans [28] and Drosophila melanogaster [29] in relation to calorie restriction (reviewed in $[30,31]$ ), the mechanisms by which this occurs have come under intense scrutiny. Sirtuin family members are involved in mechanisms such as nutrient sensing, DNA repair and the regulation of metabolism [26]. Sirt1 is upregulated by calorie restriction, responding to variations in the levels of its cofactor $\mathrm{NAD}^{+}$[32], and regulates metabolism via the deacetylation of a variety of target proteins, including notably PGC1 $\alpha$ and FoxO, through which it modulates both gluconeogenesis and glycolysis. Sirt1 also participates in the resistance to age-related apoptosis that occurs in response to stress [33].

However, Sirt1 is not alone in regulating metabolism. As would be expected, the mitochondrial sirtuins (Sirts 3, 4 and 5) are essential actors in energy metabolism. Sirt3 regulates energy homeostasis via the maintenance of basal ATP levels. Sirt $3^{-/}$mouse embryonic fibroblasts show a reduction in ATP levels and several components of the electron transport chain exhibit increased acetylation [34]. More recently it was shown that Sirt3 reversibly deacetylates the ATP synthase F1 proteins $\alpha, \beta$ and $\gamma$ and oligomycin sensitivity-conferring protein in response to nutrient or exercise-induced stress [35]. Furthermore, Sirt3 regulates the TCA cycle enzyme isocitrate dehydrogenase 2 , while deacetylation of pyruvate dehydrogenase E1 $\alpha$ subunit in response to nutrient stress allows metabolic flexibility by switching from oxidative phosphorylation to lactate production and utilization of fatty acids [36]. Sirt4 inhibits glutamine entry to the TCA cycle via the inhibition of glutamate dehydrogenase activity by ADP-ribosylation. The third mitochondrial sirtuin, Sirt5, modulates glucose metabolism via the regulation of the activities of pyruvate and succinate dehydrogenases and mitochondrial respiration, but via its deacylase (desuccinylase and demalonylase), and not deacetylase, activity [37,38] (see below).

The nuclear sirtuins, Sirts 6 and 7, have also recently been shown to have essential roles in metabolism regulation. Sirt6 is critically involved in the regulation of glucose metabolism: Sirt6 knockout (KO) mice develop a fatal hypoglycemia [39]. This sirtuin negatively regulates transcription dependent on Hif- $1 \alpha$ by deacetylating $\mathrm{H} 3 \mathrm{~K} 9$ on the promoters of its target genes, which include glucose transporter 1, lactate dehydrogenase $\mathrm{A}$ and pyruvate dehydrogenase kinase 1 [40]. The effect of the absence of Sirt6 is to promote glucose uptake and aerobic glycolysis (producing lactate), a phenotype associated with many cancer cells and known as the 'Warburg effect' [41].

In common with cancer cells, during their development in the mammalian host, schistosomes undergo a metabolic transformation similar to the Warburg effect. The infective cercariae transform into schistosomula on 


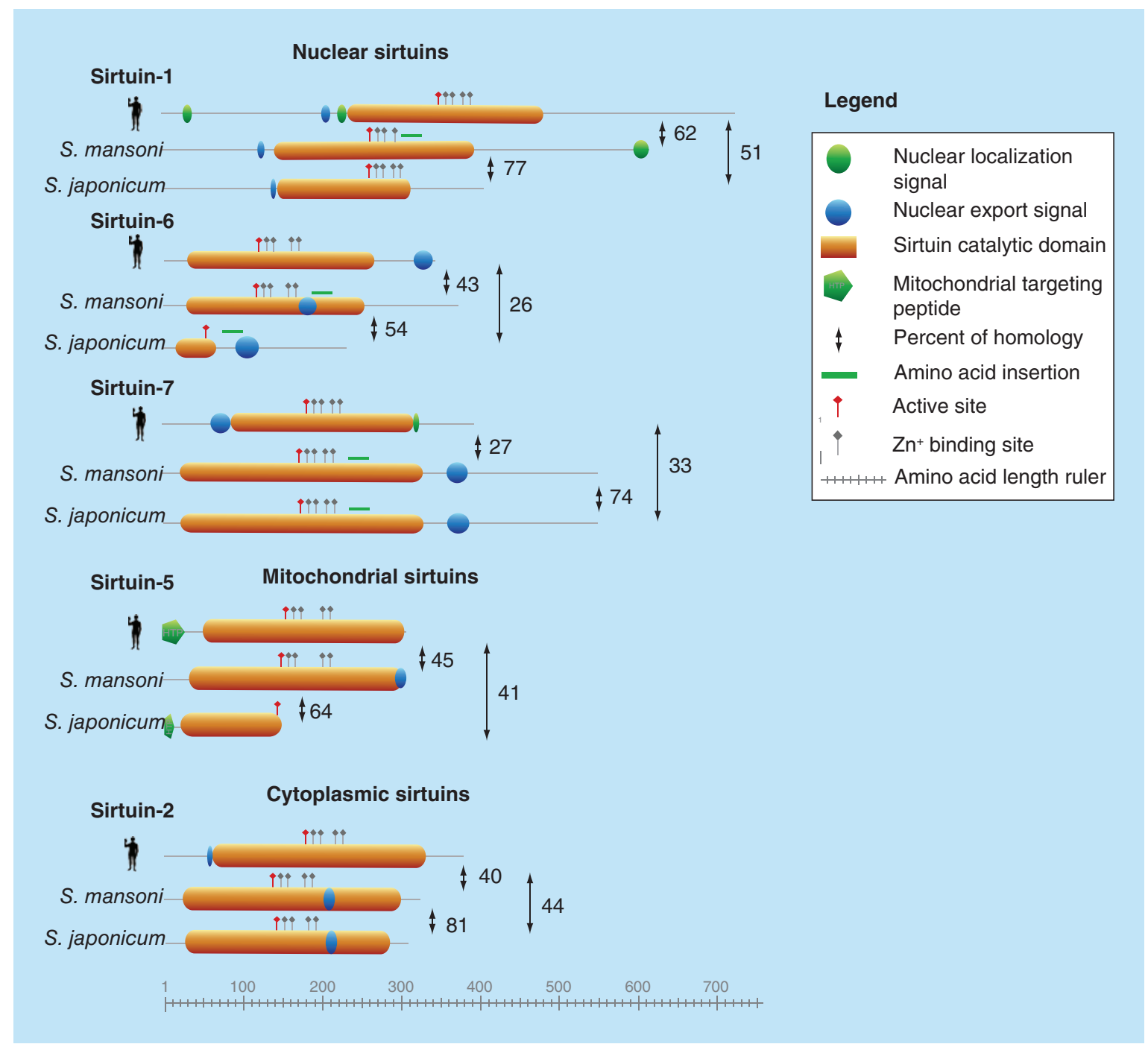

Figure 2. Comparative diagram of human and Schistosome Sirtuins. Relevant domains of Schistosome sirtuins are highlighted and compared with the homologs in humans. Drawing was performed with the online tool MyDomains (PROSITE database [25]).

penetrating the skin of a mammalian host, a biological transformation that is accompanied by both structural and physiological alterations. Notably, while the free-living cercariae have an aerobic metabolism, the schistosomula have a dominantly anaerobic metabolism, producing mainly lactate as the end-product of the degradation of glucose absorbed from the external medium [42]. This transformation seems to be entirely dependent on the presence of glucose since schistosomula maintain aerobic metabolism when cultured in the presence of only trace amounts of glucose, but switch to lactate production when glucose is added to the medium. The glucose-dependent lactate fermentation metabolism is maintained during development into adult worms. We have shown that Sirt6 transcripts are highly expressed in cercariae and newly transformed schistosomula, but are approximately 70 -fold downregulated in adult worms [16]. We are currently investigating the role of $S$. mansoni Sirt6 in this metabolic switch and in the control of schistosome glucose metabolism.

In addition to regulation of glucose metabolism, sirtuins also regulate lipid synthesis and utilization, notably shown by the use of gene KO models. Initially described as an activator of RNA polymerase I transcription [43], Sirt7 has also been shown recently to be involved in the control of lipid metabolism. Its histone (H3K18) deacetylase activity is involved in preventing fatty liver disease [44] and studies in Sirt7 KO mice show its implication in controlling liver lipid metabolism via regulation of the ubiquitin-proteasome pathway [45]. Moreover, Sirt7 deficiency also leads to mitochondrial dysfunction, including increased lactate production, via the deacetylation of GABP $\beta 1$, a regulator of nuclear-encoded mitochondrial genes [46]. Sirt1 is 
involved in controlling hepatic fatty acid utilization [47] and its ablation also leads to hepatic steatosis in vivo [48]. Similarly, Sirt6 KO mice indicate a role for lipid metabolism as they show a loss of subcutaneous fat [39] and further studies [26] reveal its role as a regulator of lowdensity lipoprotein and cholesterol. Sirt3 KO leads to the hyperacetylation of many mitochondrial proteins, and its targets include long-chain acyl-CoA dehydrogenase which is involved in fatty acid oxidation [49]. Finally, Sirt4 inhibits the activity of malonyl-CoA decarboxylase, which acts to balance mitochondrial levels of malonyl-CoA and acetyl-CoA, hence regulating the fatty acid oxidation/synthesis balance [50].

In addition to these functions in metabolism, sirtuins also have a central role in DNA repair. Moreover, these functions are linked and may be coordinated [26] since DNA damage can lead to metabolic changes that promote antioxidant defense and DNA repair [51]. Sirt4, by inhibiting mitochondrial glutamine metabolism, reroutes this amino acid to the DNA damage response [52]. Sirt1-null mouse embryos show a defective DNA damage response and a deficiency in DNA repair. A recent study [53] links $\mathrm{NAD}^{+}$depletion by the chronically active DNA repair enzyme poly-ADPribose polymerase to inactivation of Sirt1 and its target PGC-1 $\alpha$ that leads to defective mitochondria in a mouse model of Cockayne Syndrome B. Although mitochondrial Sirts 4 and 5 probably have no direct role in nuclear DNA repair, Sirt3 can locate to the nucleus under conditions of cellular stress where it modulates the expression of stress-related genes [54].

The conservation of the sirtuins in flatworms suggests that they fulfill many of the same functions as in mammals. However, some differences are evident. Notably the absence of two of the mitochondrial sirtuins, Sirt3 and 4 , in $S$. mansoni, suggests that their functions may have been taken on by other sirtuins, notably by Sirt5. In support of this possibility, at least five splicing isoforms of Sirt5 were detected as transcripts [16]. However, human Sirt5 has only low deacetylase activity [37] and the activities of the schistosome Sirt 5 isoforms remain to be characterized. In addition, the catalytic domains of both $S$. mansoni Sirt1 and 7 contain large insertions. While it is possible, it is unlikely that these affect the catalytic activity of the enzymes; insertions in the catalytic domain of $S$. mansoni HDAC8 were shown to form unstructured loops on the surface of the protein [55]. We can speculate that these insertions provide surfaces for schistosome-specific protein-protein interactions, supporting novel functions.

\section{Sirtuin structure \& catalysis}

The crystal structures have been determined for Sirt2 [56], Sirt3 [57], Sirt5 [58], Sirt6 [59] and the Sirt1 catalytic core domain [60]. All the complete structures contain a conserved catalytic core domain which is composed of a NAD ${ }^{+}$-binding Rossmann fold domain and a smaller $\mathrm{Zn}^{2+}$-binding domain that contains four highly conserved Cys residues. A model of the overall structure of sirtuins is shown in Figure 3. Schistosome sirtuins conform to this conserved overall structure, as do those of other helminths. Figure 4 shows the alignment of the catalytic core domain of Sirtl from $S$. mansoni with those from human, from the trematode C. sinensis, two cestodes (Echinococcus) and another Lophotrochozoan, the annelid C. capitata. In all cases this domain shows extensive conservation, including the four Cys residues, albeit with large insertions in the case of the schistosome and Echinococcus sequences. The fact that these insertions are found only downstream to the active site and zinc ion binding residues (Figure 2) indicates that they probably do not affect the catalytic mechanism.

The catalytic site of sirtuins is formed by a hydrophobic channel at the interface of the large and small domains (Figure 3). The bound $\mathrm{Zn}^{2+}$ ion, in contrast to that of class I, II and IV HDAC, has no catalytic role, but is involved in structural maintenance. In the sirtuin catalytic mechanism, the acetyllysine side chain of the peptide substrate is brought into close proximity with the nicotinamide ribose of $\mathrm{NAD}^{+}$. Catalysis occurs in two stages (Figure 3): nicotinamide release and the formation of a bond between the acetyl oxygen and C1', which yields an alkylimidate intermediate, followed by the formation of a second bicyclic intermediate and hydrolysis to yield the deacetylated peptide and 2'-O-acetyl-ADP-ribose.

Activities other than deacetylation have been described for sirtuins. ADP-ribosylation was described, notably in the case of Sirt4. For example, Sirt4 ADP-ribosylates GDH, suppressing its activity and repressing glutamate consumption [62]. However, for other sirtuins, the physiological significance of this activity has been called into question [63], their ADP-ribosyltransferase activity being much weaker than their deacetylase activity. More recently, Sirt 4 has been shown to exhibit a lipoamidase activity that regulates the pyruvate dehydrogenase complex [64]. Sirt5 has recently been characterized as a lysine demalonylase and desuccinylase [37] and the latter modification has a regulatory role in glucose metabolism. Furthermore Sirt6, in addition to its transcriptional activity via the deacetylation of $\mathrm{H} 3 \mathrm{~K} 9 \mathrm{ac}$, has been shown to remove a long-chain fatty acyl group from lysine, regulating the secretion of TNFa [65]. Together, these latter activities invite a reassessment of sirtuins as protein deacylases, rather than simply as deacetylases. 
(A)

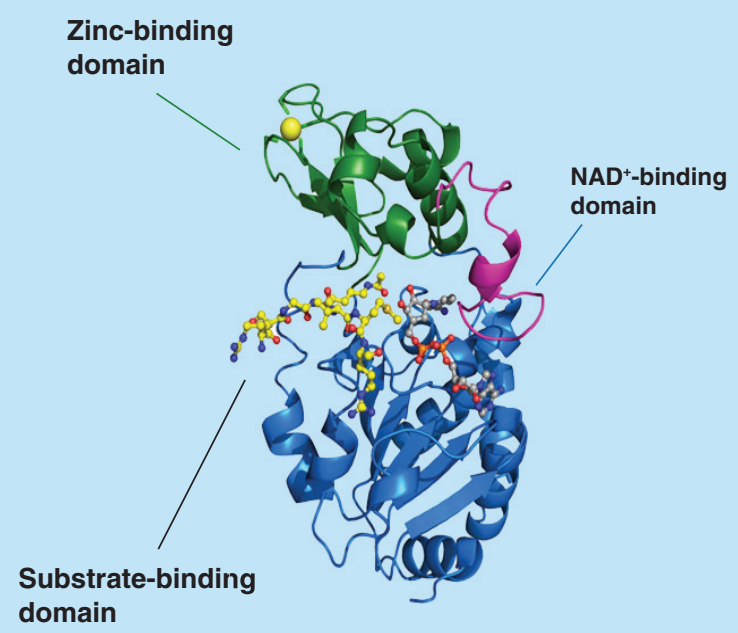

(B)

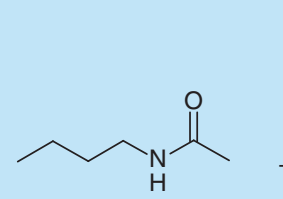

$R$<smiles>[2H]C[C@H]1O[C@@H]([n+]2cccc(C(N)=O)c2)[C@H](O)[C@@H]1O</smiles>

Acetyl-lysine

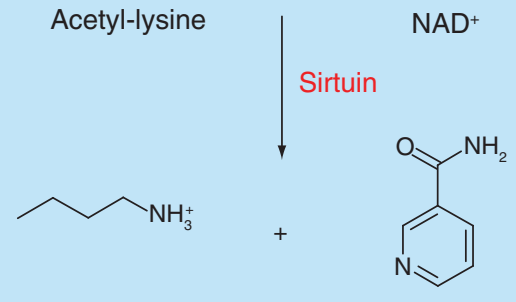

$\mathrm{NAD}^{+}$

Deacetylated-lysine

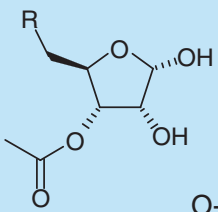

O-Ac-ADP-ribose
NAM

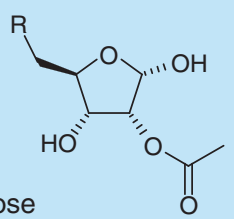

Figure 3. Sirtuin structure and catalytic mechanism. (A) Model of human Sirt1 showing the small Zn $\mathrm{n}^{2+}$-binding domain and the larger Rossman-fold domain including the NAD+-binding pocket. The acetylated peptide substrate is represented in the groove between the two domains. (B) Simplified representation of the deacetylation reaction catalyzed by sirtuins.

\section{Sirtuins, cancer \& schistosomes}

We have previously pointed out several similarities between schistosomes and tumors and this convergence is also raised by Marek et al. in this issue [66], so we will not labor the point here. Briefly, these are the cellular proliferation removed from control by the host, a degree of invisibility to the immune response and energy metabolism based on the consumption of large amounts of glucose and oxidative glycolysis producing lactate as the end-product [15]. We have already pointed out that schistosomes undergo a rapid switch from energy production based on oxidative phosphorylation in cercariae to glycolysis in schistosomula immediately after skin penetration, and this is stimulated by the presence of glucose in the external medium [42]. This metabolic switch is analogous to the Warburg effect noted in numerous tumors [41]. Sirtuins link the control of metabolism and DNA repair to tumorigenesis and it is probable that schistosome sirtuins fulfill similar functions to their human counterparts. It is therefore relevant to cite some of the ways in which sirtuins are involved in the generation or suppression of cancers as an indication of their interest as therapeutic targets.

For most of the sirtuins, roles in both the promotion and the suppression of cancers have been described, probably depending on the cellular and molecular context [67]. These considerations include possible differences in roles between mouse models and human tumors, the fact that the same sirtuins may behave differently in tumor initiation and in later stages and that they may fulfill distinct functions in different tissues [10]. Sirt1 is the most studied sirtuin in the development of cancer and its roles in metabolism, DNA damage repair and the regulation of cell survival mean that it is often an actor in tumor initiation and progression. In both breast and lung cancer Sirt1 is associated with the inactivation of hypermethylated in cancer 1 (HIC1) and of the $\mathrm{p} 53$ tumor suppressor pathway (through deacetylation of p53), decreased miR-200a and is essential for oncogenic signaling by estrogen/ estrogen receptor- $\alpha$. Further, a model [67] has been proposed in which Sirt1 has a 'bifurcated' role in cancer through genome maintenance. In normal cells it regulates high-fidelity DNA repair to promote genome stability, whereas in cancer cells it promotes low-fidelity DNA repair and cell survival. It is also crucial for the development of resistance to cancer drugs by various mechanisms including the facilitation of acquired resistance through genetic mutations [68]. However, Sirt1 is also associated with tumor suppression, notably via the deacetylation and consequent deactivation hypoxia-inducible factor $1 \alpha$, inhibiting the expression of genes targeted by this transcription factor [69].

Although counter examples exist in each case, Sirt2, Sirt3, Sirt4 and Sirt6 mainly seem to have roles in 


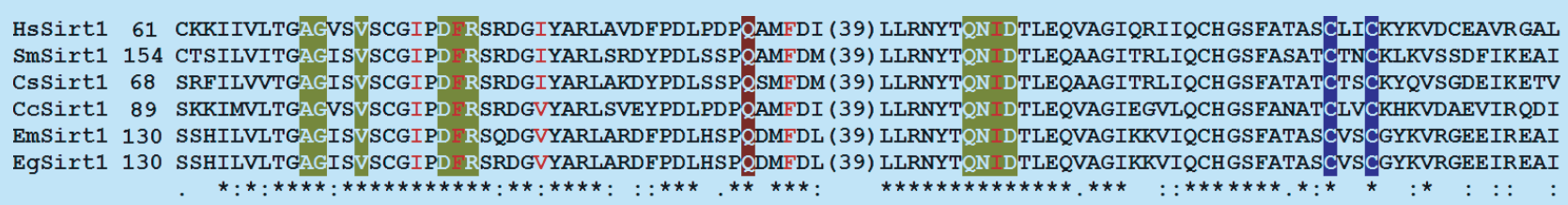

HsSirt1 197 FSQVVPRCPRCPAD----EPLAIMKPEIVFFGENLPE (14) LLIVIGSSLKVRPVALIPSSIPHEVPQITINREPLPHLHFDVELLGDCDVIINELCHRLG 302 SmSirt1 289 FTQSIPRCTNCWPS (60) ASYGVLKPDIVFFGEGLSN (14) LVLVIGSSLKVRPVSHIPNAVPRQVPQILINREPLSNHDFDVELLGDCDVIVSELCHRLG 455 CsSirt1 204 FTQSIPYCPRCHP------SFGVLKPDIVFFGEDLSS (14) LVLVIGSSLKVRPVAHIPNSIPESVPQII INREPLTNHDFDVELLGDCDVIVNELCHHLN 307 CCSirt1 234 MDQVIPHCPQCPAE----DEDAIMKPDIVFFGESLPQ (14) LLIVMGSSLKVRPVALIPNSLPANVPQVIINREPLKHLTFDVELLGDCDVIVNELCHRLG 340 EmSirt1 266 MAQRIPHCPHCRPD (30) SVHGVMKPDIVFFGEGLPA (14) LVLVMGSSLKVRPVSHIPDSVPSKVPQII INRESLSNHNFDTELLGDCDVILEHLCRQLE 402

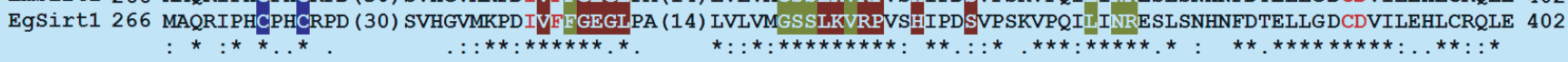

\begin{tabular}{|l|}
\hline Legend \\
Sirtuin 1 catalytic domain \\
Zinc binding \\
Acetyl-lysine peptide binding \\
NAD+ binding \\
$X \quad$ Interaction with EX527 analogue \\
\hline
\end{tabular}

Figure 4. Alignment of Sirt1 catalytic domains. Alignment of the catalytic domains of Sirt1 from human (HsSirt1), S. mansoni (SmSirt1), another trematode, C. sinensis (CsSirt1), a nonparasitic lophotrochozoan, Capitella capitata (CcSirt1) and two cestodes Echinococcus multilocularis (EmSirt1) and E. granulosus (EgSirt1). Critical residues for zinc binding, peptide substrate binding and $\mathrm{NAD}^{+}$binding are shown. Residues involved in the binding of HsSirt1 to the inhibitor Ex527 [61] are shown in red.

suppressing cancer. Sirt2 KO mice develop hepatocellular carcinoma and Sirt 2 is downregulated in human hepatocellular carcinoma and breast cancer [70]. Sirt3, through its roles in metabolism and regulating the oxidative stress response, is a mitochondrial tumor suppressor $[10,71,72]$. The expression of a single oncogene (Myc or Ras) in Sirt $3^{-1-}$ mouse embryonic fibroblasts results in in vitro transformation [71]. Moreover, deletions of Sirt3 are present in $20 \%$ of all cancers [67]. Sirt 4 has recently been suggested to be a tumor suppressor [52,73] and its expression is reduced in human cancers of the bladder, breast, colon, gastric system, ovary and thyroid [10]. Sirt6 is a tumor suppressor: it is downregulated in various human cancers and its depletion in a conditional mouse KO model leads to increases in the number, size and aggressiveness of tumors [74]. In contrast, Sirt5 and Sirt7 seem to have predominantly tumor-promoting properties. Sirt5 is overexpressed in nonsmall cell lung cancer and its knockdown represses lung cancer cell growth in vitro and in vivo [75]. Sirt7 acts via the deacetylation of $\mathrm{H} 3 \mathrm{~K} 18$, necessary for the maintenance of human cancer cell tumor phenotypes [76].

Whether sirtuins have roles as promoters or suppressors of tumors, their inhibition is likely to have profound effects on the viability of cancer cells. In the case of Sirt1 it has been argued [10] that, since heterozygous KO mice show little or no effect on development and physiology, and homozygous $\mathrm{KO}$ phenotypes are much more severe in inbred than in outbred mouse strains, Sirt1 inhibitors are unlikely to have marked side effects in humans, particularly as they would not be intended for prolonged use. Similarly, we can argue that inhibitors of parasite sirtuins, even if not completely selective vis-à-vis the human orthologues, could have significant effects on the parasite without affecting the human host. This is particularly probable since the aim is to develop novel agents that would be administered as a single dose.

\section{Sirtuin inhibitors: scaffolds \& strategies}

In view of their multiple cellular roles, notably in controlling metabolism and the DNA damage response, and their demonstrated activities as cancer promoters or repressors, sirtuins are considered as attractive epigenetic drug targets in a number of pathologies. However, despite this, only one sirtuin inhibitor, Selisistat or SEN196, is currently in clinical trials for the treatment of Huntington's disease [77]. Relatively few potent and/or selective compounds have so far been developed. Reasons for this include the pan-sirtuin inhibition often seen with sirtuin inhibitors, the fact that compounds targeting the $\mathrm{NAD}^{+}$-binding site can also interact with other enzymes that utilize $\mathrm{NAD}^{+}[78,79]$, the need for improved assays and for a better understanding of the inhibitory mechanisms of existing compounds [80]. Nevertheless, the potential for cancer therapy of sirtuin inhibitors has been demonstrated, for 


\section{Key terms}

Epigenetic drug targets: Schistosome epigenetic mechanisms are seen as a particularly sensitive target in view of the complexity of the parasite's life cycle and the tight control of gene transcription this implies. The enzymes that carry out histone modifications, including sirtuins (although these enzymes have a variety of protein substrates in addition to histones), are therefore targets of choice for the development of new drugs.

Structure-based drug discovery: The use of resolved 3D structures of sirtuins or, in some cases, models either to screen for novel modulators or to optimize existing compounds is a key to the development of drugs which will be selective for a particular sirtuin and/or for schistosome sirtuins with respect to their human orthologues.

High-throughput screening: In the particular context of antischistosome drug screening this refers to the screening for the inhibition of the enzymatic activity of recombinant schistosome sirtuins. This approach is likely to yield novel inhibitor scaffolds and warheads and, combined with molecular modelling, should facilitate the development of selective inhibitors.

instance, by the capacity of Sirtinol to induce apoptosis and autophagic cell death in the human breast cancer cell line, MCF-7 [81] and the induction of cell death and p 53 acetylation by Salermide, an inhibitor of Sirt1 and Sirt2, in the same cell line [82].

Sirtuin inhibitors and their specificities have been previously reviewed (e.g., [83,84]) and Table 1 shows a list of some of them, many of which have been tested on schistosome larvae and adult worms in culture and shown to induce apoptosis and parasite death, or marked lesions, notably to the reproductive organs [16]. Most show little selectivity when tested against Sirt1, 2 or 3 and this is a common feature of existing inhibitors. Moreover, even some of the best, recently developed inhibitors such as Ex-527 [92] and AGK2, which does show some selectivity for Sirt2 (Table 1 and [94]), show low potencies compared with typical drugs [80]. However, recent progress, both in assay development, kinetic characterization and analysis of structure-activity relationships, has meant that drug development based on sirtuin inhibitors has become more rational and effective. Structure-based drug discovery will be key to this approach, particularly for the development of parasite-selective inhibitors.

One example of new structural insights into the action of sirtuin inhibitors is provided by the analysis of the binding of Ex-527 to the Sirt 1 catalytic domain [61]. This showed that the inhibitor bound to the catalytic site, but that the binding extended into a proximal hydrophobic region. This analysis provided a basis for the development of analogues that would include extensions of the Ex-527 scaffold to reach sirtuin isoform-specific pockets [61]. Intriguingly, the residues involved in the binding of Ex-527 to human Sirt1 are well conserved in $S$. mansoni Sirt1, as well as in Sirt1 from other platyhelminth parasites (Figure 4), suggesting that this compound could form the basis for the development of parasite-specific inhibitors. An approach using tubulin-derived thioacetyllysine peptides [101] allowed the identification of a compound with an $\mathrm{IC}_{50}$ of $180 \mathrm{nM}$ for Sirt1 and a 20 -fold selectivity over Sirt2. Other structure-based approaches can now be used exploiting the specific deacylation activities of Sirt5 and Sirt6. In the case of Sirt5 the modification of residues close to the catalytic site can be targeted $[102,103]$. The establishment of an assay for Sirt5 using a small labeled succinylated lysine derivative has led to the identification of thiobarbiturates as new inhibitors of this sirtuin [104]. Sirt6 has a long, specific hydrophobic catalytic site pocket that should also allow the development of selective inhibitors [65].

The schistosome sirtuins, while showing overall conservation of essential catalytic domain residues (Figure 3 and [16]), also show significant differences. The solution of crystal structures of schistosome sirtuins bound to inhibitors would represent a significant advance for the development of selective inhibitors.

Nevertheless, the use of high-throughput screening of extensive compound libraries represents a complementary strategy that has recently been used with success to generate inhibitors of human Sirts 1, 2 and 3 that show nanomolar $\mathrm{IC}_{50}$ inhibitory values, although they are not selective [105]. It is to be expected that the application of both high-throughput and structure-based screening strategies will rapidly lead to the identification of both selective and potent sirtuin inhibitors. Indeed, in a recent study, we report the optimization of fluorescence-based assays for $S$. mansoni Sirt 2 that allowed a pilot screen with inhibitors showing $\mathrm{IC}_{50}$ values of $<50 \mu \mathrm{M}$ and docking studies rationalizing the binding of hits to the target using a homology model of the enzyme [106].

\section{Sirtuins as drug targets in parasites}

The characterization of the biological roles of the sirtuins, particularly in metabolism, DNA damage repair and cell survival, the effects of the invalidation of sirtuin genes and/or transcripts in mouse models and the activities of sirtuin inhibitors all underline the status of sirtuins as drug targets in human pathologies, most obviously in cancer. But what about parasites, in general, and schistosomes in particular? A recent review [13] gives a thorough overview of the structure and functions of sirtuins from protozoan parasites and their potential as therapeutic targets, particularly in Plasmodium falciparum and Leishmania. Although the $\mathrm{KO}$ of either of the $P$. falciparum sirtuin-encoding genes shows that neither is essential 


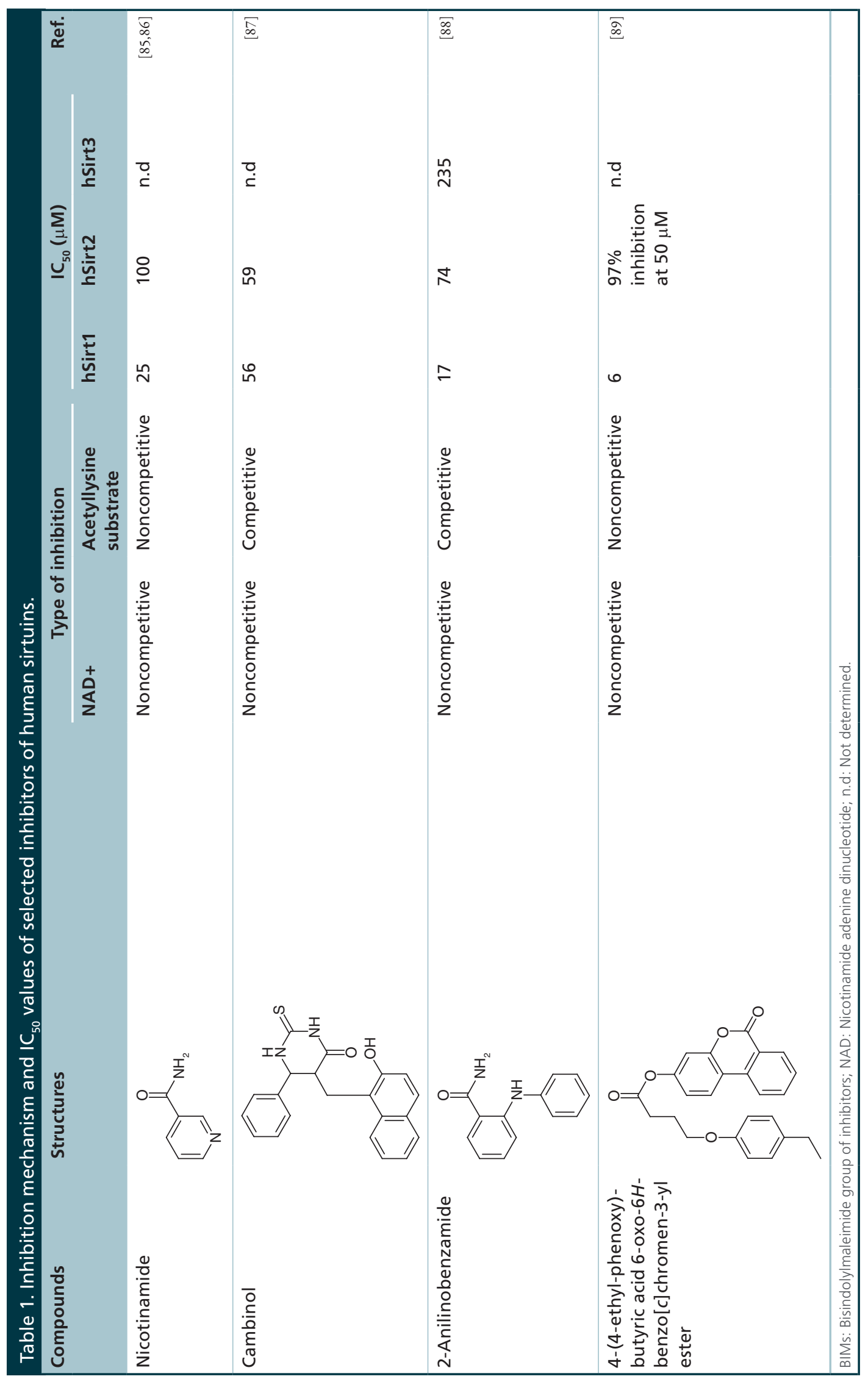


Review Lancelot, Cabezas-Cruz, Caby et al.

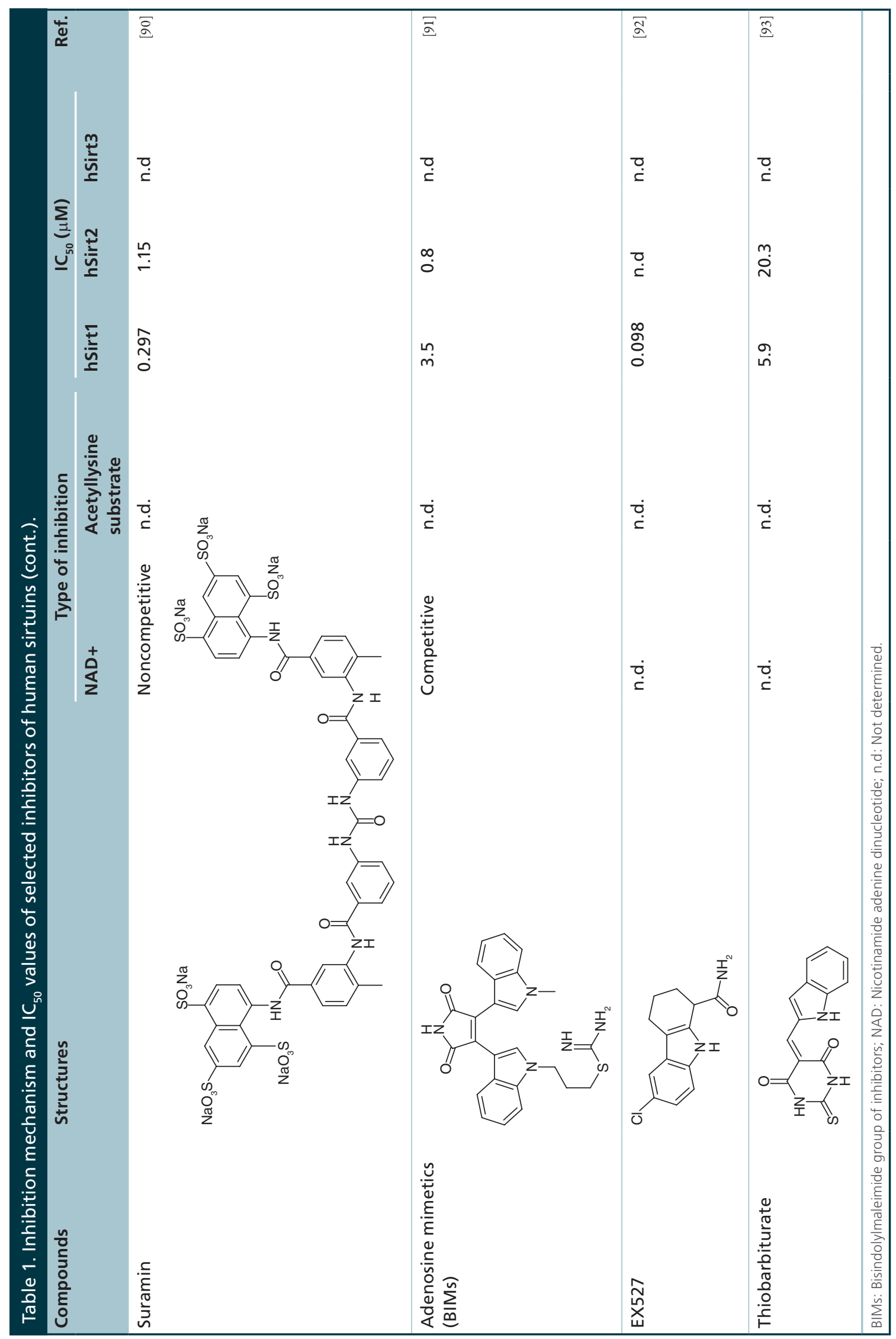




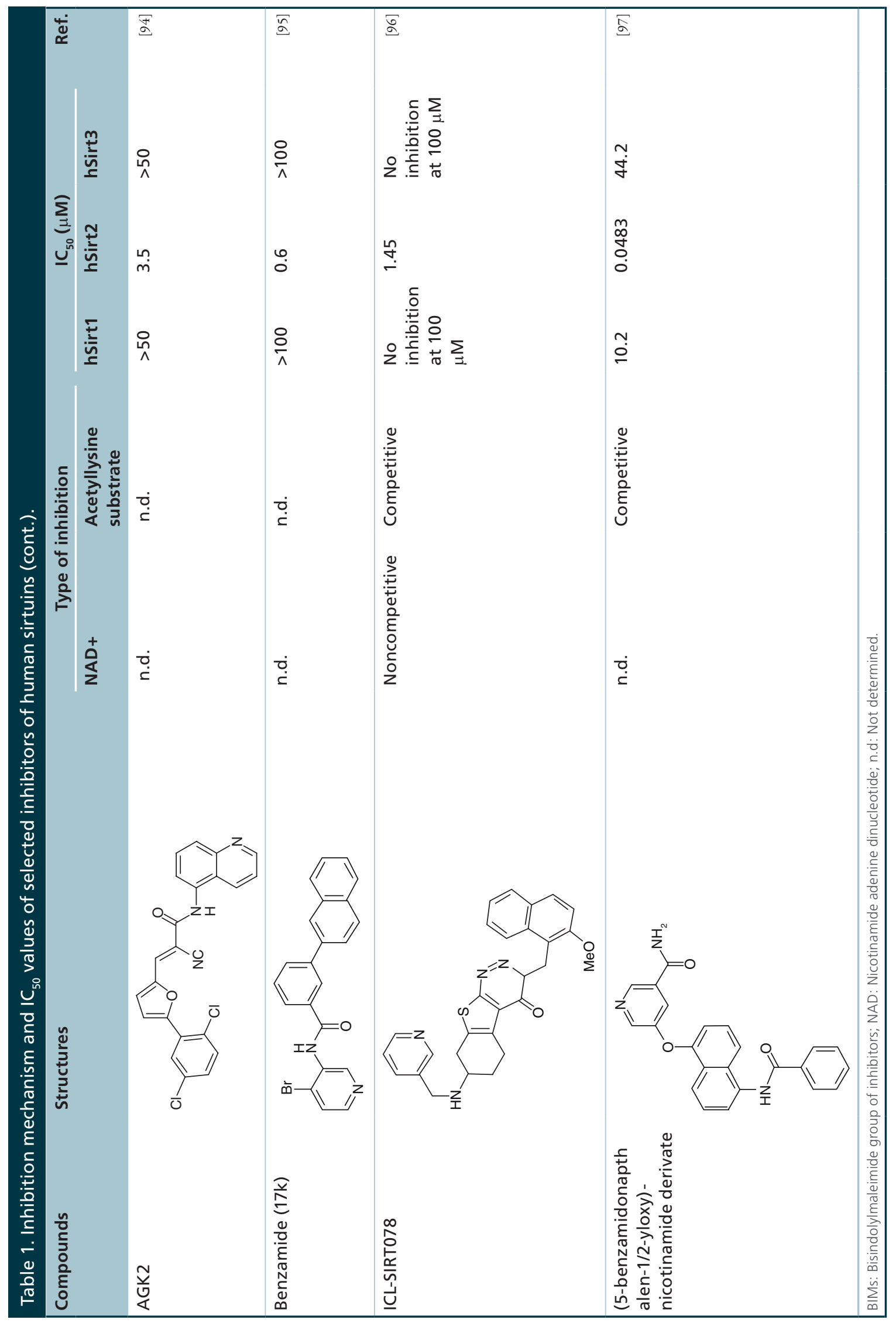




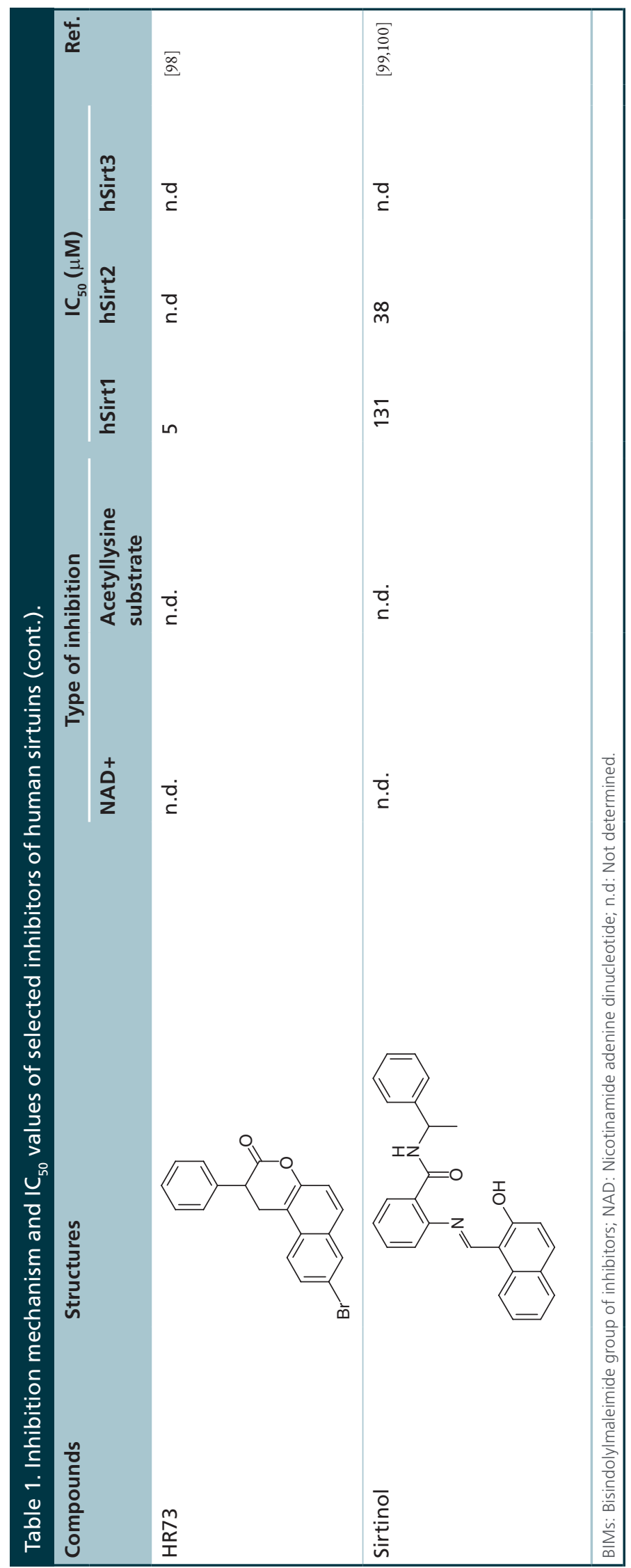




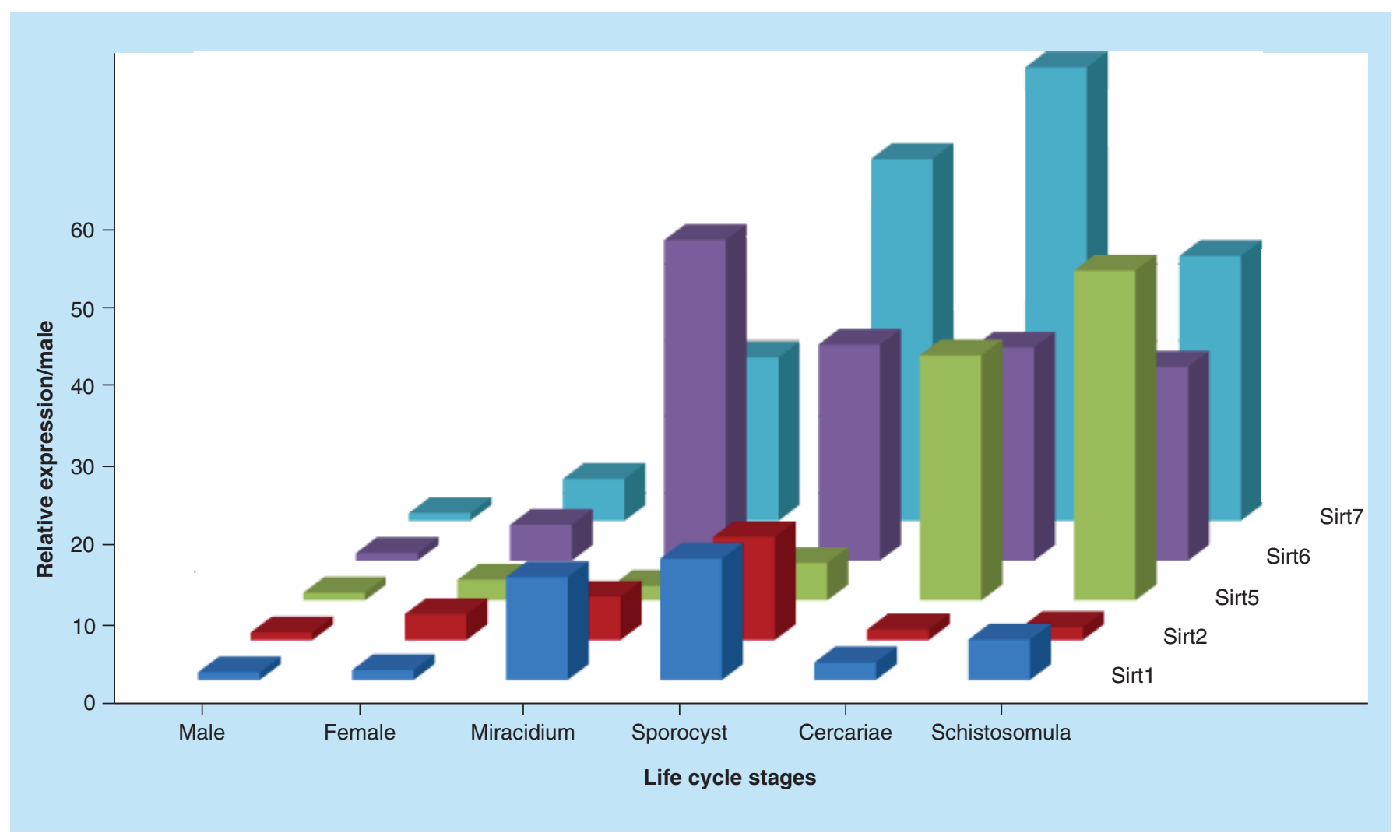

Figure 5. Transcriptional regulation of sirtuins during the life cycle of Schistosoma mansoni. Levels of transcripts of the five $S$. mansoni sirtuins were measured in different life-cycle stages by quantitative RT-PCR: male and female adult worms, miracidia, sporocysts, cercariae and schistosomula. Values are expressed relative to the levels in adult male worms. Adapted with permission from [16].

for parasite proliferation $[107,108]$, the roles of both sirtuins in the epigenetic control of the expression of the var genes, encoding variable surface antigens involved in the evasion of the host immune response, have been established. In the case of Leishmania, the sirtuin LiSIR2RP1 appears to be essential since LiSir2rp $1^{-1-}$ parasites could only be obtained when the enzyme was expressed episomally [109]. Following the demonstration that Sirtinol was able to inhibit the growth of Leishmania infantum axenic amastigotes [110], a more specific inhibitor, bisnaphthalimidopropyl has been developed that is selective for the parasite sirtuin LiSIR2RP1 compared with human Sirt1 [111]. Sirtuins are therefore targets of choice for antiprotozoan parasite drug development.

In S. mansoni, sirtuins are expressed at all life-cycle stages, but there are wide variations in the levels of transcripts, particularly in the case of the nuclear sirtuins, Sirt6 and Sirt7 (Figure 5), which are highly expressed in larval stages, whereas the nucleo-cytosolic sirtuins, Sirt1 and Sirt2 show less variation. The knockdown of Sirt1 transcripts causes marked changes to female worm reproductive organs [16]) including an increase in mature oocytes in the ovaries. These changes are similar to those produced by treatment with the sirtuin inhibitor
Salermide, although these were more pronounced, possibly since Sirt2 would also be inhibited in this case. Moreover, several sirtuin inhibitors induced the unpairing of worm pairs in culture and significant reductions in egg laying [16]. Although we have no data as yet concerning the effects of sirtuin transcript knockdown in the schistosomula larvae, sirtuin inhibitors, particularly Salermide, readily induce apoptosis and death of the larvae at 10 and $20 \mu \mathrm{M}$ concentrations. The relative abundance of transcripts of the mitochondrial sirtuin Sirt 5 and the two nuclear sirtuins Sirt 6 and Sirt7 in the infective larval stages [16] suggests that these may have crucial roles during schistosome development and therefore represent sensitive targets for chemotherapy.

\section{Future perspective}

Sirtuins are crucial regulators of cellular processes, two of which, energy metabolism and the DNA repair response, determine their potential importance as therapeutic targets. However, in the case of cancer, sirtuins can either promote or repress tumor development, and either inhibition or activation of their activity might be therapeutic or detrimental. This means that for cancer therapy it is important that the sirtuin target and the clinical situation 
should be well defined. The need for individual sirtuinselective inhibitors is manifest [10]. In the case of parasitic diseases, in contrast, it is evident that the inhibition of sirtuins is sufficient to cause detrimental effects to the parasite and that species selectivity and/or enhanced bioavailability to the parasitic organism is most important.

The differences in the catalytic domains of schistosome sirtuins compared with their human counterparts are likely to be sufficient to allow the development of selective inhibitors, but this requires verification by molecular modeling and, where possible, $\mathrm{x}$-ray crystallographic studies. The study of the class I HDAC8 from $S$. mansoni [55] showed that the crystal structure was indispensable in order to detect all the differences in the architecture of the catalytic pocket of the enzyme, and this is likely to be even more critical for sirtuins in which two domains, the $\mathrm{NAD}^{+}$-binding domain and the peptide substrate-binding domain, have to be considered. However, high-throughput screening of recombinant enzymes can also allow the detection of inhibitors with novel scaffolds and warheads that are selective. We are currently pursuing strategy involving both approaches, in the context of an European Commission-funded project [112], aimed at developing new drugs against Neglected Parasitic Diseases, including schistosomiasis.

\section{Financial \& competing interests disclosure}

This work and the authors of this manuscript have been supported by funding from the European Union's Seventh Framework Programme for research, technological development and demonstration under grant agreement nos. 241865 (SEtTReND) and 602080 (A-ParaDDisE). CR, MM and RJP are supported by institutional funds from the Centre National de la Recherche Scientifique (CNRS), the Institut National de la Santé et de la Recherche Médicale (INSERM), the Université de Strasbourg and the Université de Lille 2, the French Infrastructure for Integrated Structural Biology (FRISBI; ANR-10INSB-05-01) and by Instruct (ESFRI). MJ thanks the Deutsche Forschungsgemeinschaft for funding (DFG, Project P10 within RTG1976). The authors have no other relevant affiliations or financial involvement with any organization or entity with a financial interest in or financial conflict with the subject matter or materials discussed in the manuscript apart from those disclosed.

No writing assistance was utilized in the production of this manuscript.

\section{Executive summary}

- The current reliance on a single drug, praziquantel, for the treatment of schistosomiasis means that the development of new drugs is a priority.

- Sirtuins, which are $\mathrm{NAD}^{+}$-dependent protein deacylases, are promising drug targets in a variety of pathologies.

- Schistosomes express orthologues of five of the seven sirtuins present in humans and lack two of the mitochondrial isoforms.

- Schistosome sirtuins have conserved catalytic domains, but show sufficient differences to allow the development of selective inhibitors.

- Sirtuins have diverse and crucial biological roles, particularly in the control of metabolism and the DNA repair response.

- Sirtuins have either cancer promoting or repressing roles and sirtuin-based chemotherapy can aim either at inhibition or enhancement of sirtuin enzyme activity.

- Sirtuin inhibitors inhibit the growth of protozoan parasites and induce apoptosis and death of schistosome larvae, while inducing massive modifications to adult worm reproductive organs.

- Sirtuins are valid therapeutic targets for the development of novel drugs against parasites and, in particular, against schistosomes.

\section{References}

Papers of special note have been highlighted as:

• of interest; $\bullet \bullet$ of considerable interest.

1 Fenwick A, Webster JP, Bosque-Oliva E et al. The Schistosomiasis Control Initiative (SCI): rationale, development and implementation from 2002-2008. Parasitology 136(13), 1719-1730 (2009).

2 Doenhoff MJ, Cioli D, Utzinger J. Praziquantel: mechanisms of action, resistance and new derivatives for schistosomiasis. Curr. Opin. Infect. Dis. 21(6), 659-667 (2008).

3 Berriman M, Haas B, Loverde PT et al. The genome of the blood fluke Schistosoma mansoni. Nature 460 (7253), 352-358 (2009).
4 Zhou Y, Zheng H, Chen X et al. The Schistosoma japonicum genome reveals features of host-parasite interplay. Nature 460(7253), 345-351(2009).

5 Young ND, Jex AR, Li B et al. Whole-genome sequence of Schistosoma haematobium. Nat. Genet. 44(2), 221-225 (2012).

6 Cabezas-Cruz A, Lancelot J, Caby S, Oliveira G, Pierce RJ. Epigenetic control of gene function in schistosomes: a source of therapeutic targets? Front. Genet. 5 , 317 (2014).

- Reviews current knowledge of epigenetic mechanisms in schistosomes and evaluates their potential as therapeutic targets.

7 Herranz D, Munoz-Martin M, Canamero M et al. Sirt1 improves healthy ageing and protects from metabolic syndrome-associated cancer. Nat. Commun. 1, 3 (2010). 
8 Kaeberlein M. Lessons on longevity from budding yeast. Nature 464(7288), 513-519 (2010).

9 Libert S, Guarente L. Metabolic and neuropsychiatric effects of calorie restriction and sirtuins. Annu. Rev. Physiol. 10, 669-684 (2013).

10 Yuan H, Su L, Chen WY. The emerging and diverse roles of sirtuins in cancer: a clinical perspective. Onco Targets Ther. 6, 1399-1416 (2013).

-• A comprehensive and recent review of the roles of human sirtuins as cancer promoters or repressors and the potential for therapeutic intervention.

11 Greiss S, Gartner A. Sirtuin/Sir2 phylogeny, evolutionary considerations and structural conservation. Mol. Cells 28(5), 407-415 (2009).

- Revises the phylogeny of the sirtuins and highlights the differences in the complement of sirtuins in different species.

12 Costantini S, Sharma A, Raucci R, Costantini M, Autiero I, Colonna G. Genealogy of an ancient protein family: the sirtuins, a family of disordered members. BMC Evol. Biol. 13, 1-19 (2013).

13 Religa A, Waters A. Sirtuins of parasitic protozoa: in search of function(s). Mol. Biochem. Parasitol. 185(2), 71-88 (2012).

- Explores the roles of sirtuins in parasitic protozoa and emphasizes their interest as drug targets.

14 Zheng W. Sirtuins as emerging anti-parasitic targets. Eur. J. Med. Chem. 59, 132-140 (2013).

15 Pierce R, Dubois-Abdesselem F, Lancelot J, Andrade L, Oliveira G. Targeting schistosome histone modifying enzymes for drug development. Curr. Pharm. Des. 18(24), 3567-3578 (2012).

16 Lancelot J, Caby S, Dubois-Abdesselem F et al. Schistosoma mansoni sirtuins: characterization and potential as chemotherapeutic targets. PLoS Negl. Trop. Dis. 7, e2428 (2013).

- $\quad$ Characterizes the sirtuins of $S$. mansoni and shows that sirtuin inhibitors induce apoptosis and death of the parasites, emphasizing their potential as anti-schistosomal drugs.

17 Frye RA. Phylogenetic classification of prokaryotic and eukaryotic Sir2-like proteins. Biochem. Biophys. Res. Commun. 273(2), 793-798 (2000).

18 Cavalier-Smith T. "The origin of Fungi and pseudofungi". In: Evolutionary biology of the fungi: symposium of the british mycological society. Rayner ADM, Brasier CM, Moore D (Eds). Cambridge Univ. Press, Cambridge, UK, 339-353 (1987).

19 Michishita E, Park JY, Burneskis JM, Barrett JC, Horikawa I. Evolutionarily conserved and nonconserved cellular localizationos and functions of human SIRT proteins. Mol. Biol. Cell. 16(10), 4623-4635 (2005).

20 North BJ, Verdin E. Interphase nuleo-cytoplasmic shuttling and localization of SIRT2 during mitosis. PLoS ONE 2(8), e784, (2007).

21 Vaquero A, Scher MB, Lee DH et al. SirT2 is a histone deacetylase with preference for histone H4Lys 16 during mitosis. Genes Dev. 20(10), 1256-1261 (2006).

22 Scher MB, Vaquero A, Reinberg D. SirT3 is a nuclear NAD+-dependent histone deacetylase that translocates to the mitochondria upon cellular stress. Genes Dev. 21(8), 920-928, (2007).

23 Park J, Chen Y, Tishkoff DX et al. SIRT5 -mediated lysine desuccinylation impacts diverse metabolic pathways. Mol. Cell. 50(6), 919-930 (2013).

24 Matsushita N, Yonashiro R, Ogata Y et al. Distinct regulation of mitochondrial localization and stability of two human Sirt5 isoforms. Genes Cells 16(2), 190-202 (2011).

25 Sigrist CJ, de Castro E, Cerutti $\mathrm{L}$ et al. New and continuing developments at PROSITE. Nucl. Acids Res. 41, D344-D347 (2013).

26 Choi J-E, Mostoslavsky R. Sirtuins, metabolism and DNA repair. Curr. Opin. Genet. Dev. 26, 24-32 (2014).

-. Comprehensive and recent review of the crucial roles of sirtuins in the control of metabolism, and the link with their role in the DNA repair response.

27 Kaeberlein M, McVey M, Guarente L. The SIR2/3/4 complex and SIR 2 alone promote longevity in Saccharomyces cerevisiae by two different mechanisms. Genes Dev. 13(19), 2570-2580 (1999).

28 Tissenbaum HA, Guarente L. Increased dosage of a sir-2 gene extends lifespan in Caenorhabditis elegans. Nature 410(6825), 227-230 (2001).

29 Wood JG, Rogina B, Lavu S et al. Sirtuin activators mimic caloric restriction and delay ageing in metazoans. Nature 430(7000), 686-689 (2004).

30 Westphal CH, Dipp MA, Guarente L. A therapeutic role for sirtuins in diseases of aging? Trends Biochem. Sci. 32(12), 555-560 (2007).

31 Guarente L. Calorie restriction and sirtuins revisited. Genes Dev. 27(19), 2072-2085 (2013).

32 Cantò C, Auwerx J. NAD ${ }^{+}$as a signaling molecule modulating metabolism. Cold Spring Harb. Symp. Quant. Biol. 76, 291-298 (2011).

33 Brooks CL, Gu W. How does SIRT1 affect metabolism, senescence and cancer? Nat. Rev. Cancer 9(2), 123-128 (2009).

34 Ahn BH, Kim HS, Song $S$ et al. A role for mitochondrial deacetylase Sirt 3 in regulating energy homeostasis. Proc. Natl Acad. Sci. USA 105(38), 4447-4452 (2008).

35 Vassilopoulos A, Pennington JD, Andresson T et al. SIRT3 deacetylates ATP synthase FA complex proteins in response to nutrient- and exercise-induced stress. Antioxid. Redox Signal. 21(4), 551-564 (2014).

36 Jing E, O'Neill BT, Rardin MJ et al. Sirt3 regulates metabolic flexibility of skeletal muscle through reversible enzymatic deacetylation. Diabetes 62 (10), 3404-3417 (2013).

$37 \mathrm{Du}$ J, Zhou Y, Su X et al. Sirt5 is a NAD-dependent protein lysine demalonylase and desuccinylase. Science 334(6057), 806-809 (2011).

38 Park J, Chen Y, Tishkoff DX et al. Sirt5-mediated lysine desuccinylation impacts diverse metabolic pathways. Mol. Cell 50(6), 919-930 (2013).

39 Mostoslavsky R, Chua KF, Lombard DB et al. Genomic instability and aging-like phenotype in the absence of mammalian SIRT6. Cell 124(2), 315-329 (2006). 
40 Zhong L, Urso AD, Toiber D et al. The histone deacetylase Sirt6 regulates glucose homeostasis via Hif1alpha. Cell 140(2), 280-293 (2010).

41 Warburg O. On the origin of cancer cells. Science 123(3191), 309-314 (1956).

42 Horemans AMC, Tielens AGM, van den Bergh SG. The reversible effect on the energy metabolism of Schistosoma mansoni cercariae and schistosomula. Mol. Biochem. Parasitol. 51(1), 73-80 (1992).

43 Ford E, Voit R, Liszt G, Magin C, Grummt I, Guarente L. Mammalian Sir2 homolog SIRT7 is an activator of RNA polymerase I transcription. Genes Dev. 20(9), 1075-1080 (2006)

44 Shin J, He M, Liu Y et al. Sirt7 represses Myc activity to suppress ER stress and prevent fatty liver disease. Cell Rep. 5(3), 654-665 (2013).

45 Yohizawa T, Karim MF, Sato Y et al. SIRT7 controls hepatic lipid metabolism by regulating the ubiquitin proteasome pathway. Cell Metab. 19(4), 712-721 (2014).

46 Ryu D, Jo YS, Lo Sasso G et al. A SIRT7-dependent acetylation switch of GABP $\beta 1$ controls mitochondrial function. Cell Metab. 20(5), 856-869 (2014).

47 Rodgers JT, Puigserver P. Fasting-dependent glucose and lipid metabolic response through hepatic sirtuin 1. Proc. Natl Acad. Sci. USA 104(31), 12861-12866 (2007)

48 Purushotham A, Schug TT, Xu Q, Surapureddi S, Guo X, Li X. Hepatocyte-specific deletion of SIRT1 alters fatty acid metabolism and results in hepatic steatosis and inflammation. Cell Metab. 9(4), 327-338 (2009).

49 Hirschey MD, Shimazo T, Goetzman E et al. SIRT3 regulates mitochondrial fatty-acid oxidation by reversible enzyme deacetylation. Nature 464(7285), 121-125 (2010).

50 Laurent G, German NJ, Saha AK et al. SIRT4 coordinates the balance between lipid synthesis and catabolism by repressing malonyl CoA decarboxylase. Mol. Cell 50(5), 686-698 (2013).

51 Cosentino C, Grieco D, Costanzo V. ATM activates the pentose phosphate pathway promoting anti-oxidant defence and DNA repair. EMBO J. 30(3), 546-555 (2010).

52 Jeong SM, Xiao C, Finley LW et al. SIRT4 has tumorsuppressive activity and regulates the cellular metabolic response to DNA damage by inhibiting mitochondrial glutamate metabolism. Cancer Cell 23 (4), 450-463 (2013).

53 Scheibye-Knudsen M, Mitchell SJ, Fang EF et al. A high fat diet and $\mathrm{NAD}(+)$ activate Sirt 1 to rescue premature aging in Cockayne syndrome. Cell Metab. 20(5), 840-855 (2014).

54 Iwahara T, Bonasio R, Narendra V, Reinberg D. SIRT3 functions in the nucleus in the control of stress-related gene expression. Mol. Cell. Biol. 32(24), 5022-5034 (2012).

55 Marek M, Kannan S, Hauser AT et al. Structural basis for the inhibition of histone deacetylase 8 (HDAC8), a key epigenetic player in the blood fluke Schistosoma mansoni. PLoS Pathog. 9(9), e1003645 (2013).

-• Validates schistosome HDAC8 as an epigenetic drug target and shows that schistosome-specific changes in the catalytic pocket should allow the development of selective inhibitors as drug leads.
56 Finnin MS, Donigian JR, Pavletich NP. Structure of the histone deacetylase SIRT2. Nat. Struct. Biol. 8(7), 621-625 (2001).

57 Jin L, Wei W, Jiang Y et al. Crystal structures of human SIRT3 displaying substrate-induced conformational changes. J. Biol. Chem. 284(36), 24394-24405 (2009).

58 Schuetz A, Min J, Antoshenko T et al. Structural basis of inhibition of the human NAD ${ }^{+}$-dependent deacetylase SIRT5 by suramin. Structure 15(3), 377-389 (2007).

59 Pan PW, Feldman JL, Devries MK, Dong A, Edwards AM, Denu JM. Structure and biochemical functions of SIRT6. J. Biol. Chem. 286(16), 14575-14587 (2011).

60 Zhao X, Allison D, Condon B et al. The $2.5 \AA$ crystal structure of the SIRT1 catalytic domain bound to nicotinamide adenine dinucleotide $\left(\mathrm{NAD}^{+}\right)$and an indole (EX527 analogue) reveals a novel mechanism of histone deacetylase inhibition. J. Med. Chem. 56(3), 963-969 (2013).

61 Gertz M, Fischer F, Nguyen GT et al. Ex-527 inhibits Sirtuins by exploiting their unique $\mathrm{NAD}^{+}$-dependent deacetylation mechanism. Proc. Natl Acad. Sci. USA 110(30), E2772-2781 (2013).

62 Haigis MC, Mostoslavsky R, Haigis KM et al. SIRT4 inhibits glutamate dehydrogenase and opposes the effects of calorie restriction in pancreatic beta cells. Cell 126(5), 941-954 (2006).

63 Du J, Jiang H, Lin H. Investigating the ADPribosyltransferase activity of sirtuins with NAD analogs and ${ }^{32}$ P-NAD. Biochemistry 48(13), 2878-2890 (2009).

64 Mathias RA, Greco TM, Oberstein A et al. Sirtuin 4 is a lipoamidase regulating pyruvate dehydrogenase complex activity. Cell 159(7), 1615-1625 (2014).

65 Jiang H, Khan S, Wang Y et al. SIRT6 regulates TNF-alpha secretion through hydrolysis of long-chain fatty acyl lysine. Nature 496(7443), 110-113 (2013).

66 Marek M, Oliveira G, Pierce RJ, Sippl W, Jung M, Romier C. Zinc-dependent erasers of protein acetylation in schistosomes: on the track of HDAC inhibitors as new antiparasitic drugs. Future Med. Chem. (2014) (In Press).

67 Roth M, Chen WY. Sorting out functions of sirtuins in cancer. Oncogene 33(13), 1609-1620 (2014).

68 Wang Z, Chen W. Emerging roles of SIRT1 in cancer drug resistance. Genes Cancer 4(3-4), 82-90 (2013).

69 Lim JH, Lee YM, Chun YS, Chen J, Kim JE, Park JW. Sirtuin 1 modulates cellular responses to hypoxia by deacetylating hypoxia-inducible factor 1alpha. Mol. Cell 38(6), 864-878 (2010).

70 Kim HS, Vassilopoulos A, Wang RH et al. SIRT2 maintains genome integrity and suppresses tumorigenesis through regulating APC/C activity. Cancer Cell 20 (4), 487-499 (2011).

71 Kim HS, Patel K, Muldoon-Jacobs K et al. SIRT3 is a mitochondria-localized tumor suppressor required for maintenance of mitochondrial integrity and metabolism during stress. Cancer Cell 17(1), 41-52 (2010).

72 Finley LW, Carracedo A, Lee J et al. SIRT3 opposes reprogramming of cancer cell metabolism through HIF1 alpha destabilization. Cancer Cell 19(3), 416-428 (2011). 
73 Csibi A, Fendt SM, Li C et al. The mTORC1 pathway stimulates glutamine metabolism and cell proliferation by repressing SIRT4. Cell 153(4), 840-854 (2013).

74 Sebastián C, Zwaans BM, Silberman DM et al. The histone deacetylase SIRT6 is a tumor suppressor that controls cancer metabolism. Cell 151(6), 1185-1199 (2012).

75 Lu W, Zuo Y, Feng Y, Zhang M. SIRT5 facilitates cancer cell growth and drug resistance in non-small cell lung cancer. Tumour Biol. 35(11), 10699-10705 (2014).

76 Barber MF, Michishita-Kioi E, Xi Y et al. SIRT7 links H3K18 deacetylation to maintenance of oncogenic transformation. Nature 487(7405), 114-118 (2012).

77 Arrowsmith CH, Bountra C, Fish PV, Lee K, Schapira M. Epigenetic protein families: a new frontier for drug discovery. Nat. Rev. Drug Discov. 11(5), 384-400 (2012).

78 Wall KA, Klis M, Kornet J et al. Inhibition of the intrinsic $\mathrm{NAD}^{+}$glycohydrolase activity of CD38 by carbocyclic NAD analogues. Biochem. J. 335(Pt 3) 631-636 (1998).

79 Slama JT, Simmons AM. Inhibition of NAD glycohydrolase and ADP-ribosyltransferases by carbocyclic analogues of oxidized nicotinamide adenine dinucleotide. Biochemistry 28(19), 7688-7694 (1989).

80 Schutkowski M, Fischer F, Roessler C, Steegborn C. New assays and approaches for discovery and design of Sirtuin modulators. Expert Opin. Drug Discov. 9(2), 183-199 (2014).

81 Wang J, Kim TH, Ahn MY et al. Sirtinol, a class III HDAC inhibitor, induces apoptotic and autophagic cell death in MCF-7 human breast cancer cells. Int. J. Oncol. 41(3), 1101-1109 (2012).

82 Lara E, Mai A, Calvanese V et al. Salermide, a sirtuin inhibitor with a strong cancer-specific proapoptotic effect. Oncogene 28(6), 781-791 (2009).

83 Neugebauer RC, Sippl W, Jung M. Inhibitors of NAD ${ }^{+}$dependent histone deacetylases (sirtuins). Curr. Pharm. Des. 14(6), 562-573 (2008).

84 Cen Y. Sirtuin inhibitors: the approach to affinity and selectivity. Biochim. Biophys. Acta 1804(8), 1635-1644 (2010).

85 Sauve AA, Schramm VL. Sir2 regulation by nicotinamide results from switching between base exchange and deacetylation chemistry. Biochemistry 42(19), 9249-9256 (2003).

86 Schmidt MT, Smith BC, Jackson MD, Denu JM. Coenzyme specificity of Sir2 protein deacetylases: implications for physiological regulation. J. Biol. Chem. 279(38), 4012240129 (2004).

87 Heltweg B, Gatbonton T, Schuler AD et al. Antitumor activity of a small molecule inhibitor of human silent information regulator 2 enzymes. Cancer Res. 66(8), 4368-4377 (2006).

88 Suzuki T, Imai K, Nakagawa H, Miyata N. 2-Anilobenzamides as SIRT inhibitors. ChemMedChem 1(10), 1059-1062 (2006).

89 Sanders BD, Jackson B, Brent M et al. Identification and characterization of novel sirtuin inhibitor scaffolds. Bioorg Med. Chem. 17(19), 7031-7041 (2009).
90 Trapp J, Meier R, Hongwiset D, Kassak MU, Sippl W, Jung M. Structure-activity studies on suramin analogues as inhibitors of $\mathrm{NAD}^{+}$-dependent histone deacetylases (sirtuins). ChemMedChem 2(10), 1419-1434 (2007).

91 Trapp J, Jochum A, Meier R et al. Adenosine mimetics as inhibitors of $\mathrm{NAD}^{+}$-dependent histone deacetylases, from kinase to sirtuin inhibition. J. Med. Chem. 49(25), 7307-7316 (2006).

92 Napper AD, Hixon J, McDonagh T et al. Discovery of indoles as potent and selective inhibitors of the deacetylase SIRT1. J. Med. Chem. 48(25), 8045-8054 (2005).

93 Uciechowska U, Schemies J, Neugebauer RC et al. Thiobarbiturates as sirtuin inhibitors: virtual screening, freeenergy calculations and biological testing. ChemMedChem 3(12), 1965-1976 (2008).

94 Outeiro TF, Kontopoulos E, Altmann SM et al. Sirtuin 2 inhibitors rescue alpha-synuclein-mediated toxicity in models of Parkinson's disease. Science 317(5837), 516-519 (2007).

95 Sakai T, Matsumoto Y, Ishikawa $\mathrm{M}$ et al. Design, synthesis and structure-activity relationship studies of novel sirtuin 2(SIRT2) inhibitors with a benzamide skeleton. Bioorg. Med. Chem. 23(2), 328-339 (2015).

96 Di Fruschia P, Zacharioudakis E, Liu C et al. The discovery of a highly selective 5,6,7,8-tetrahydrobenzo [4,5] thieno[2,3-d]pyrimidin- $4(3 \mathrm{H})$-one SIRT2 inhibitor that is neuroprotective in and in vitro Parkinson's disease model. ChemMedChem 10(1), 69-82 (2015).

97 Cui H, Kamal Z, Ai T et al. Discovery of potent and selective sirtuin 2 (SIRT2) inhibitors using a fragment-based approach. J. Med. Chem. 57(20), 8340-8357 (2014).

98 Hirao M, Posakony J, Nelson M et al. Identification of selective inhibitors of $\mathrm{NAD}^{+}$-dependent deacetylases using phenotypic screens in yeast. J. Biol. Chem. 278(52), 52773-52782 (2003).

99 Grozinger CM, Chao ED, Blackwell HE, Moazed D, Schreiber SL. Identification of a class of small molecule inhibitors of the sirtuin family of NAD-dependent deacetylases by phenotypic screening. J. Biol. Chem. 276(42), 38837-38843 (2001)

100 Mai A, Massa S, Lavu S et al. Design, synthesis and biological evaluation of sirtinol analogues as class III histone/ protein deacetylase (Sirtuin) inhibitors. J. Med. Chem. 48(24), 7789-7795 (2005).

101 Kiviranta PH, Suuronen T, Wallen EA et al. N(epsilon)thioacetyl-lysine-containing tri-, tetra- and pentapeptides as SIRT1 and SIRT2 inhibitors. J. Med. Chem. 52(7), 2153-2156 (2009).

102 Suenkel B, Fischer F, Steegborn C. Inhibition of the human deacylase Sirtuin 5 by the indole GW5074. Bioorg. Med. Chem. Lett. 23(1), 143-146 (2013).

103 Roessler C, Nowak T, Pannek M et al. Chemical probing of the human sirtuin 5 active site reveals its substrate acyl specificity and peptide-based inhibitors. Angew. Chem. Int. Ed. Engl. 53(40), 10728-10732 (2014).

104 Maurer B, Rumpf T, Scharfe M et al. Inhibitors of the $\mathrm{NAD}(+)$-dependent protein desuccinylase and demalonylase Sirt5. ACS Med. Chem. Lett. 3(12), 1050-1053 (2012). 
105 Disch JS, Evindar G, Chiu CH et al. Discovery of thieno[3,2-d]pyrimidine-6-carboxamides as potent inhibitors of SIRT1, SIRT2 and SIRT3. J. Med. Chem. 56(9), 3666-3679 (2013).

106 Schiedel M, Marek M, Lancelot J et al. Fluorescence-based screening assays for the $\mathrm{NAD}^{+}$-dependent histone deacetylase smSirt2 from Schistosoma mansoni. J. Biomol. Screen. 20(1), 112-121 (2015).

- Describes assay methods developed for the high-throughput screening of a recombinant schistosome sirtuin.

107 Duraisingh MT, Voss TS, Marty AJ et al. Heterochromatin silencing and locus repositioning linked to regulation of virulence genes in Plasmodium falciparum. Cell 121(1), 13-24 (2005).

108 Tonkin CJ, Carret CK, Duraisingh MT et al. Sir2 paralogues cooperate to regulate virulence genes and antigenic variation in Plasmodium falciparum. PLoS Biol. 7(4), e84 (2009).
109 Vergnes B, Sereno D, Tavares J et al. Targeted disruption of cytosolic SIR2 deacetylase discloses its essential role in Leishmania survival and proliferation. Gene 363, 85-96 (2005).

110 Vergnes B, Vanhille L, Ouaissi A, Sereno D. Stage-specific antileishmanial activity of an inhibitor of SIR2 histone deacetylase. Acta Trop. 94(2), 107-115 (2005).

111 Tavares J, Ouaissi A, Kong Thoo Lin P et al. Bisnaphthalimidopropyl derivatives as inhibitors of Leishmania SIR2 related protein 1. ChemMedChem 5(1), 140-147 (2010).

112 Pierce RJ. Anti-parasitic drug discovery. Pan Europ. Networks Sci. Technol. 11, 192-193 (2014). 